\title{
Marsupialização como tratamento inicial conservador do cisto odontogênico calcificante: relato de caso
}

\author{
Marsupialization as conservative initial treatment for calcifying odontogenic cyst: case report \\ Marsupialización como tratamiento inicial conservador del quiste odontogénico calcificante: \\ reporte de caso
}

\author{
Darllon Manuel Borges Souza \\ ORCID: https://orcid.org/0000-0002-9858-6921 \\ Centro Universitário Tiradentes, Brasil \\ E-mail: darllonmanuelsouzada@hotmail.com \\ Danillo Alves Dantas de Lima \\ ORCID: https://orcid.org/0000-0002-6178-473X \\ Centro Universitário Tiradentes, Brasil \\ E-mail: danilloalves947@gmail.com \\ Lucas Mariz de Menezes Torres \\ ORCID: https://orcid.org/0000-0001-7543-7715 \\ Centro Universitário Tiradentes, Brasil \\ E-mail:1marizdemenezes@gmail.com \\ Marielly dos Anjos Ferreira da Silva \\ ORCID: https://orcid.org/0000-0001-8599-6385 \\ Centro Universitário Tiradentes, Brasil \\ E-mail: anjosmarielly@gmail.com \\ Beatriz Nogueira dos Santos \\ ORCID: https://orcid.org/0000-0003-1748-9167 \\ Centro Universitário Cesmac, Brasil \\ E-mail: beatriznogueira44@hotmsil.com \\ Lucya Giselle Costa Moreira \\ ORCID: https://orcid.org/0000-0002-3658-8163 \\ Centro Universitário Tiradentes, Brasil \\ E-mail: lucya_giselle@hotmail.com \\ Janaína Soares da Silva \\ ORCID: https://orcid.org/0000-0002-0249-7327 \\ Centro Universitário Tiradentes, Brasil \\ E-mail: janainasooaressilva@hotmail.com \\ João Augusto Vianna Goulart-Filho \\ ORCID: https://orcid.org/0000-0001-8591-7798 \\ Faculdade São Leopoldo Mandic, Brasil \\ E-mail: joao.goulart@ souunit.com.br \\ Marcus Antônio Brêda Júnior \\ ORCID: https://orcid.org/0000-0001-6515-472X \\ Universidade de São Paulo, Brasil \\ E-mail: marcusbredajr@gmail.com \\ José Ricardo Mikami \\ ORCID: https://orcid.org/0000-0002-2300-5117 \\ Universidade de São Paulo, Brasil \\ E-mail: ricardomikami@hotmail.com
}

\begin{abstract}
Resumo
Introdução: $O$ cisto odontogênico calcificante (COC) surge na cavidade bucal como uma tumefação de crescimento lento, normalmente indolor, com prevalência na região anterior de maxila e mandíbula sem predileção por gênero, acometendo idade média de 30 anos. Objetivo: O objetivo desse trabalho é relatar a importância do diagnóstico junto a abordagem cirúrgica minimamente invasiva para patologias de grandes dimensões. Relato de caso: Paciente de 14 anos de idade, com queixa de aumento de volume progressivo do lado esquerdo da face próximo ao nariz, há cerca de 01 (um) ano, sendo submetido a marsupialização e biópsia incisional como abordagem cirúrgica inicial para o tratamento. Discussão: O relato de caso possui características semelhantes ao encontrado na literatura sobre COC até o presente momento, concordando com diagnóstico, tratamento e acompanhamento pós-cirúrgico. Conclusão: A marsupialização realizada mostrou regressão do tamanho da lesão e formação de áreas de calcificação da lesão, trazendo benefícios para o paciente.
\end{abstract}

Palavras-chave: Cisto Odontogênico Calcificante; Cisto odontogênico; Descompressão cirúrgica. 


\begin{abstract}
Introduction: The calcifying odontogenic cyst (COC) appears in the oral cavity as a slow growing swelling, usually painless, with prevalence in the anterior region of the maxilla and mandible without gender predilection, affecting an average age of 30 years. Objective: The objective of this paper is to report the importance of diagnosis with the minimally invasive surgical approach for large pathologies. Case report: A 14-year-old patient, complaining of progressive swelling on the left side of the face close to the nose, for about 01 (one) year, undergoing marsupialization and incisional biopsy as the initial surgical approach for treatment . Discussion: The case report has characteristics similar to those found in the literature on COC to date, in agreement with diagnosis, treatment and post-surgical follow-up. Conclusion: The marsupialization performed showed regression of the lesion size and formation of calcification areas in the lesion, bringing benefits to the patient. Keywords: Calcifying Odontogenic Cyst; Odontogenic Cyst; Surgical Decompression.
\end{abstract}

Keywords: Odontogenic Cyst Calcifying; Odontogenic cyst; Decompression, surgical.

\title{
Resumen
}

Introducción: El quiste odontogénico calcificante (AOC) aparece en la cavidad oral como una tumefacción de crecimiento lento, generalmente indoloro, con predominio en la región anterior del maxilar y mandíbula sin predilección de género, afectando una edad promedio de 30 años. Objetivo: El objetivo de este trabajo es reportar la importancia del diagnóstico con abordaje quirúrgico mínimamente invasivo para patologías grandes. Caso clínico: Paciente de 14 años, que se queja de hinchazón progresiva en el lado izquierdo de la cara cerca de la nariz, durante aproximadamente 01 (un) año, siendo sometido a marsupialización y biopsia incisional como abordaje quirúrgico inicial para el tratamiento. Discusión: El caso clínico presenta características similares a las encontradas en la literatura sobre AOC hasta la fecha, de acuerdo con el diagnóstico, tratamiento y seguimiento posquirúrgico. Conclusión: La marsupialización realizada mostró regresión del tamaño de la lesión y formación de áreas de calcificación en la lesión, trayendo beneficios al paciente.

Palabras clave: Quiste Odontogénico Calcificante; Quiste odontogénico; Descompresión quirúrgica.

\section{Introdução}

Gorlin, em 1962, foi o primeiro a descrever o cisto odontogênico calcificante (COC). Essa lesão é considerada rara, pois representa apenas $0,3 \%$ das lesões bucais e 2\% dos cistos e tumores dentários (Medeiros et.al., 2007). A Organização Mundial de saúde (OMS) em 1971 reconhece o COC como uma entidade clínica. A maioria apresenta lesões císticas e não neoplásicas, embora já existam relatos de lesões sólidas, o que requer uma subdivisão (Moura et al., 2019; Olmedo-Cueva et al, 2020).

O COC surge na cavidade bucal como uma tumefação de crescimento lento, normalmente indolor, com prevalência na região anterior de maxila e mandíbula, comumente iniciada da região medular do osso. Não tem predileção por gênero e normalmente acomete adultos com prevalência entre a segunda e sexta décadas de vida, tendo média de 30 anos de idade (Moura et al., 2019; Cavalcante et al., 2019). Radiograficamente tem uma característica de lesão radiolúcida unilocular ou multilocular, margens indefinidas ou circunscritas, associadas ou não a dentes irrompidos, porém está mais relacionada a dentes não irrompidos. Essa lesão pode ser classificada como central ou periférica, de acordo com a radiografia (Cavalcante et al., 2019; Pontes et.al., 2010).

Algumas característrica do cisto lembram muito o ameloblastoma, com revestimento epitelial por células basais cuboidais e/ou colunares e sua camada voltada à superfície com células idênticas ao retículo estrelado do órgão do esmalte. O COC também possui células epiteliais com ausência de núcleo, eosinófilas, denominadas de células fantasmas (Cavalcante et al., 2019; Azambuja et al., 2005; Cancio et.al., 2005).

Na maioria dos casos é observado uma expansão e afinamento da cortical óssea envolvida, podendo apresentar pontos de fenestração óssea pela lesão. A chance de recidiva da lesão é bastante considerável, o que permite fazer um acompanhamento clínico e radiográfico de rotina trimestral inicialmente (Azambuja et al., 2005; Carvalhosa et.al. 2005).

A forma de tratamento para o cisto de Gorlin segue os padrões da lesão, assim a enucleação com curetagem da lesão intraóssea e a marsupialização, sendo essas para a variante central, já para a variante periférica e neoplásica a excisão é o método de tratamento satisfatório (Azambuja et al., 2005; Kusama et al., 2005; Trejo \& Bello, 1999). 
O objetivo deste estudo é relatar um caso clínico de um cisto odontogênico calcificante em maxila com envolvimento de dente incluso em um adolescente de 14 anos.

\section{Metodologia}

Foi realizado todo o procedimento de anamnese, exame físico, exame de imagem, que contribuiu para o diagnóstico, em seguida foi explicado todo o procedimento a ser feito, esclarecendo todos cuidados pós-operatório, risco e benefícios do procedimento. Após o procedimento de biopsia o material foi encaminhado ao histopatológico para análise, onde foi diagnosticado com Cisto Ontogênico Calcificante. O genitor do paciente assinou o TCLE (termo de consentimento livre e esclarecido), que consta a autorização para divulgação dos dados do paciente para fins acadêmico. O presente artigo se trata de um estudo analítico descritivo o qual foi fundamentado nos conceitos da técnica de Pereira, et.al (2018). O procedimento foi realizado no Hospital Geral Do Estado. Não se tornando necessário passar pelo comitê de ética.

\section{Relato de Caso}

Paciente de 14 anos de idade, gênero masculino, feoderma, foi encaminhado devido a queixa de aumento de volume progressivo intra e extrabucal do lado esquerdo da face próximo ao nariz, há cerca de 01 (um) ano. Na história médica não relatou doenças sistêmicas, hábitos ou histórico familiar relevantes.

No exame físico extrabucal o paciente apresentava aumento de volume na região de espaço canino esquerdo, de consistência firme e indolor, delimitado e sem alteração de cor da pele da região envolvida (Figura 1). Ao exame intrabucal foi observado dente 23 ausente na arcada dentária aumento de volume em fundo de vestíbulo da mesial do dente 21 à distal do dente 25, com periferia de consistência endurecida e centro mais amolecido, compatível com expansão/absorção da cortical óssea vestibular, sem alteração de cor da mucosa na região (Figura 2).

Figura 1: Exame extrabucal evidenciando assimetria e aumento de volume.

A) Vista frontal;

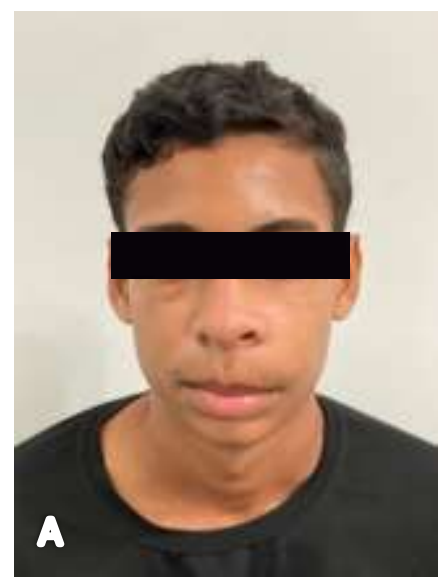

B) Vista axial.

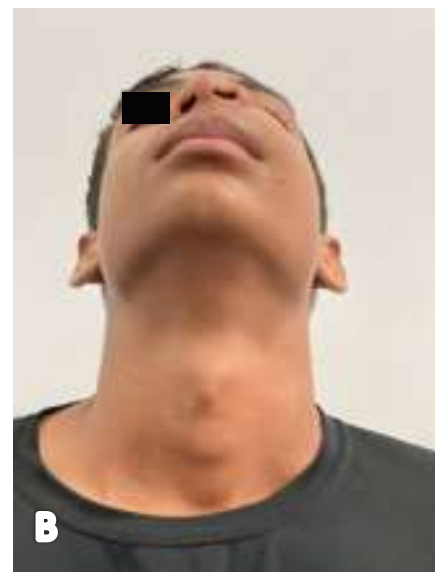

Fonte: Autores. 
Figura 2: Aspecto clínico do aumento de volume intra-oral. A) Vista intrabucal com o paciente em oclusão.B) Vista oclusal Intrabucal com o paciente em desoclusão.

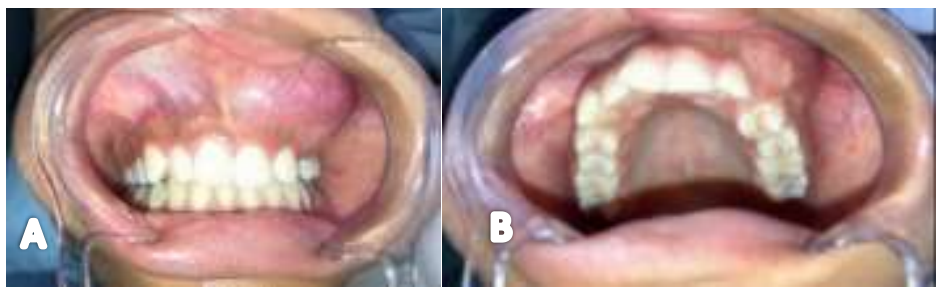

Fonte: Autores.

Nas imagens da tomografia computadorizada observou-se área hipodensa em região anterior de maxila esquerda, com cerca de $3 \times 2 \mathrm{~cm}$ de diâmetro, formato regular, limites definidos, com envolvimento do elemento 23 incluso e deslocado em direção superior próximo ao seio maxilar. Havia destruição parcial da cortical vestibular da maxila esquerda, mas com cortical palatina preservada (Figura 3) e (Figura 4).

Figura 3: Tomografia computadorizada (multislice) em corte para partes moles. A) vista sargital; B) vista coronal; C) vista axial.

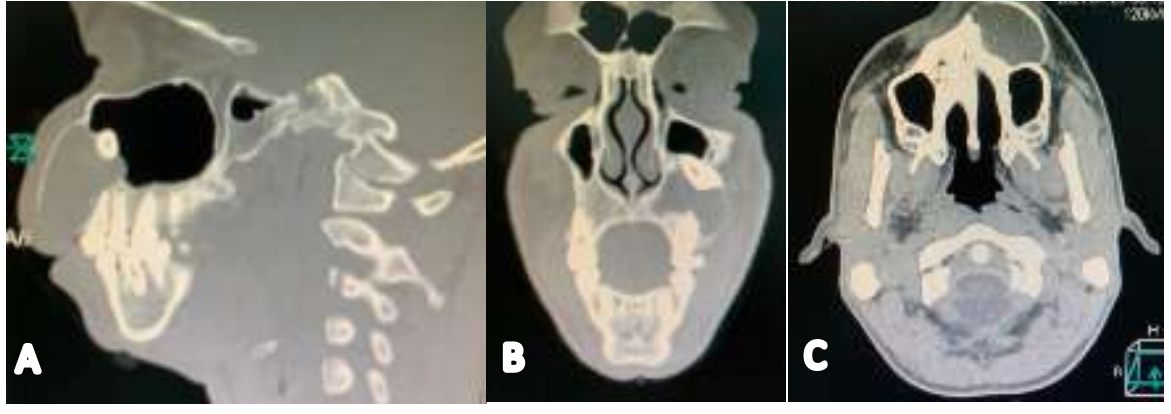

Fonte: Autores.

Figura 4: Reconstrução 3D da tomografia computadorizada do tipo multislice. A) vista axial; B) vista sagital; C) vista frontal.

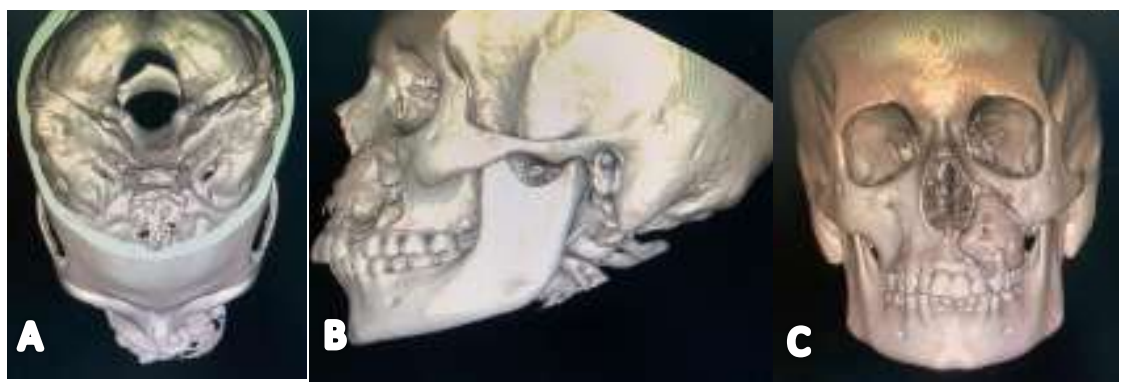

Fonte: Autores.

Para complementação do diagnóstico, realizou-se procedimento sob anestesia local iniciado por punção aspirativa e obtenção de líquido citrino (Figura 5) seguido de biópsia incisional. Também foi decidido realizar marsupialização no mesmo tempo cirúrgico, suturando-se a cápsula da lesão à mucosa, para promover uma descompressão (Figura 6). 
Figura 5: Punção aspirativa positiva com Obtenção de líquido citrino.

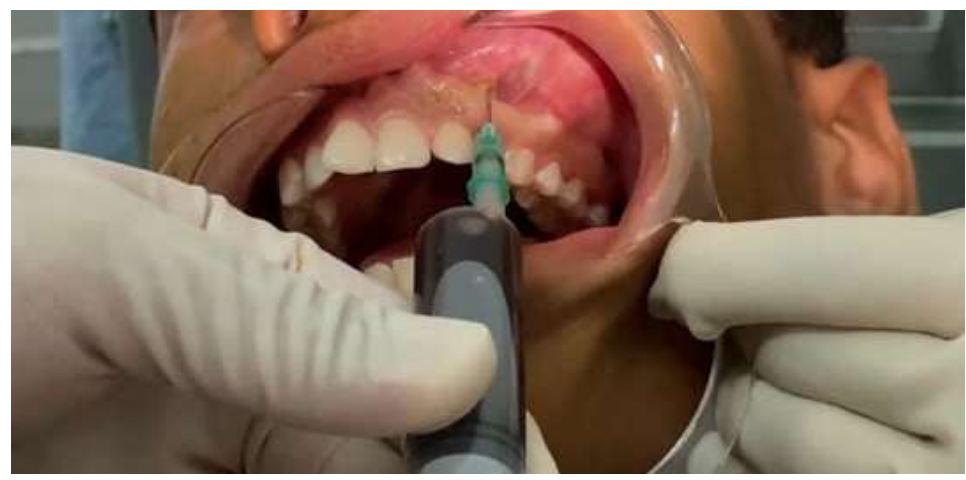

Fonte: Autores.

Figura 6: Demonstrando o procedimento de biopsia e inicial de descompressão. A) Identificação da capsula cística tracionando-a com fio de sutura de nylon; B) Peça cirúrgica que foi encaminhada par biopsia; C) Sutura da capsula cística na mucosa bucal com nylon 5-0, conhecido como marsupialização.

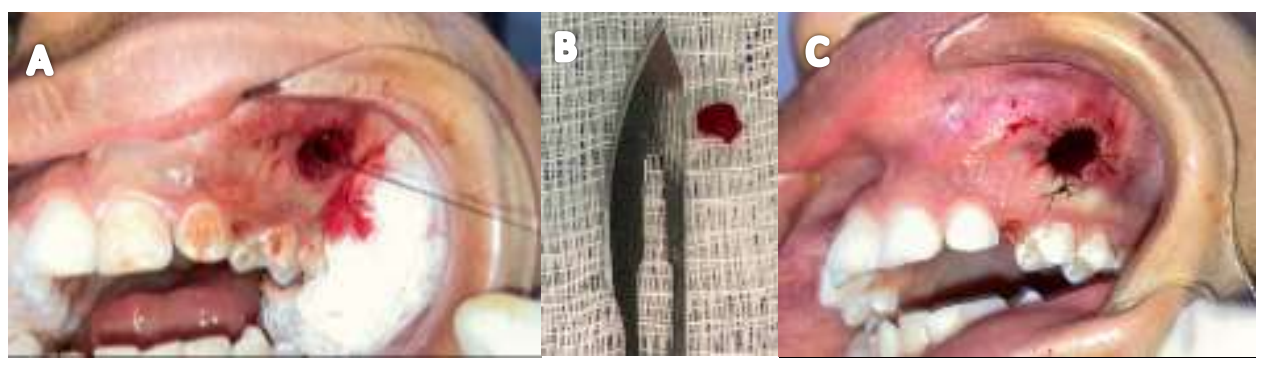

Fonte: Autores.

O paciente foi orientado quanto à limpeza da cavidade patológica com $20 \mathrm{ml}$ de soro fisiológico $0,9 \%$ por meio de irrigação com seringa tipo Luer e agulha $25 \times 7 \mathrm{~mm}$. A prescrição pós operatória consistiu de amoxicilina $(500 \mathrm{mg} 8 / 8 \mathrm{~h}$ por sete dias), ibuprofeno (600mg 8/8h por três dias) e dipirona (500mg 6/6h por dois dias).

O material da biópsia foi enviado para exame anatomopatológico onde foi observada lesão cística de natureza odontogênica caracterizada por epitélio estratificado de espessura variável mostrando em seus trechos mais espessos, camada basal com células colunares baixas, semelhante à pré-ameloblastos, camada intermediária de arranjo frouxo semelhante ao retículo estrelado do órgão do esmalte, e em seu interior grupos de células anucleadas de citoplasma amplo e de coloração eusinofílicas (conhecidas como células fantasmas). Estas últimas células formam aglomerado na forma de uma massa de material amorfo e eusinofílico que, em diversos segmentos do espécime, sofre calcificação e assume coloração basófílica. Calcificações na forma de pequenos grânulos sobre o epitélio cístico e calcificações individuais das células-fantasma também podem ser identificados nos cortes histológicos. A cápsula cística é composta por tecido conjuntivo frouxo, exibindo numerosos vasos sanguíneos de pequenos calibres e, de permeio, um discreto infiltrado inflamatório mononuclear. Áreas hemorrágicas extensas, restos de epitélio odontogênico na forma de pequenos agrupamentos e fileiras celulares, além de vasos congestos, completam o quadro histológico com o diagnóstico de cisto odontogênico calcificante (Figura 7). 
Figura 7: Imagem do exame histopatológico. A) Demonstra o revestimento da cavidade cística com células epiteliais ameloblastosas, com uma camada basal colunar, representado pela seta preta. B) Presença de material dentinóide eosinofílico em setas pretas, rodeados de células fantasmas exibidos em setas verdes.
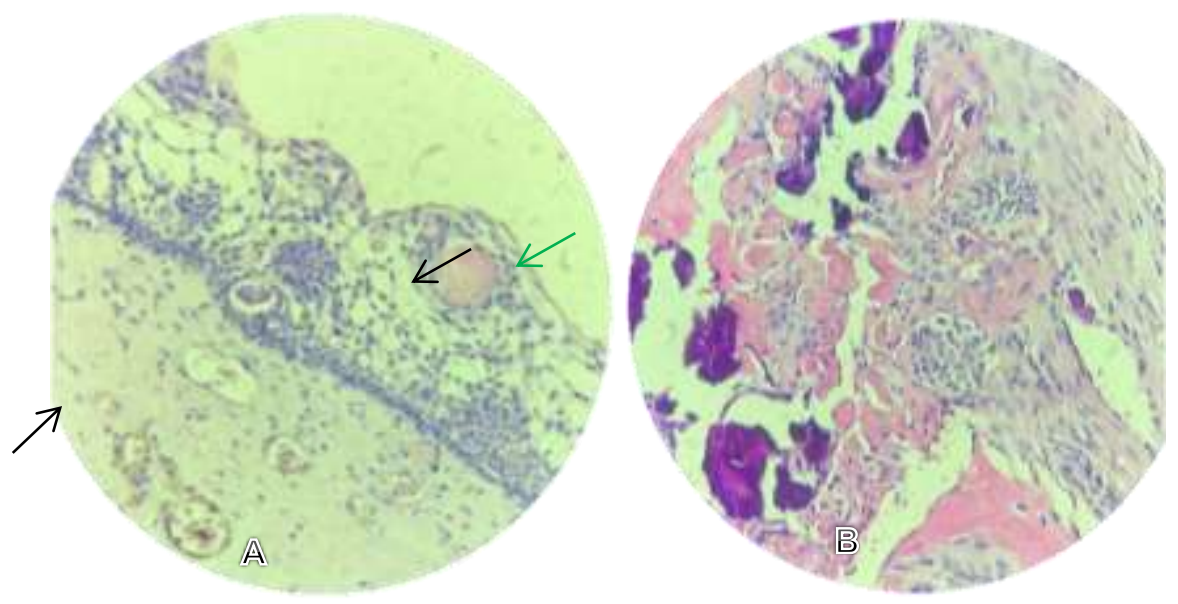

Fonte: Autores.

Após 3 (Três) meses de acompanhamento, foi solicitado uma nova tomografia, onde foram observadas imagens hiperdensas em seu interior compatíveis com áreas de calcificação onde não foi evidenciado na primeira tomografia, sendo essa calcificação característica da lesão (Figura 8).

Figura 8: Tomografia computadorizada de cortes para partes moles de 3 meses após a marsupialização. A) Corte sagital mostrando focos de calcificação no interior do cisto; B) Corte coronal mostrando focos de calcificação no interior do cisto;

C) Corte axial mostrando focos de calcificação no interior do cisto.

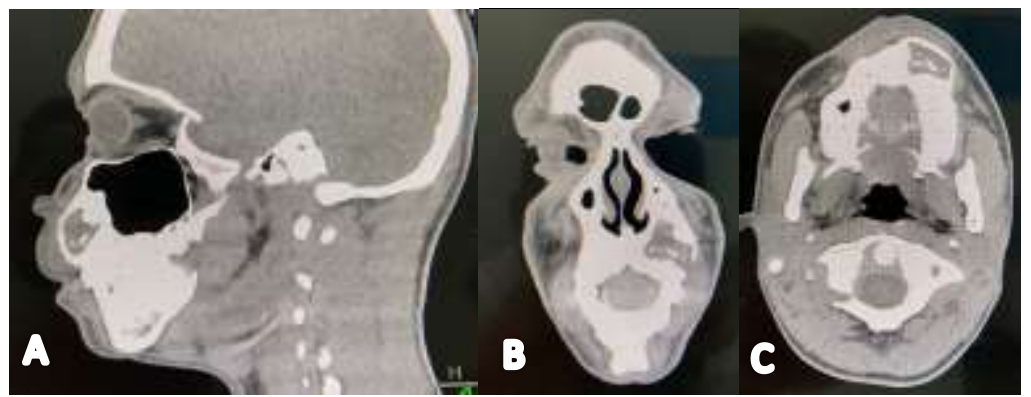

Fonte: Autores.

O paciente continua em acompanhamento clínico para controle da evolução da marsupialização e determinação final do tratamento.

\section{Discussão}

O COC apresenta prevalência por idade, sendo essa em duas faixas etárias mais comuns, entre 10-19 anos e 60-69 anos e média de 30 anos, sem predileção por gênero ou etnia (Daniels, 2004; Neville, 2016). O caso descrito anteriormente encontra-se dentro dessa margem relatada na literatura, enquadrado no primeiro período de maior incidência de lesões.

A localização do cisto está relacionada com sua época de aparecimento, tendo a mesma proporção de ocorrência entre maxila e mandíbula e área pertencente ao dente canino como a mais afetada (Moreli et al., 2002), sendo subdivididos em lesões centrais, cuja identificação é estabelecida na segunda década de vida e as de surgimento periférico que são comumente 
encontradas após a quinta década (MC Cowan, 1982). A lesão em questão obedece a característica de lesão do tipo central com dente incluso envolvido, dentro das quais se evidenciam quatros casos aqui presentes.

O diagnóstico para correto tratamento está condicionado à soma das informações clínicas observadas com os exames radiográficos complementares, sendo através desses possível observar lesão radiolúcida unilocular ou multilocular com presença de calcificações irregulares que variam de manchas pequenas a grandes massas espalhadas em toda extensão da lesão. As placas corticais ósseas são delgadas e expandidas podendo ocorrer rompimento da mesma, ocasionando reabsorção radicular de dentes adjacentes e ainda associação com algum dente não irrompido (Moreli et al., 2002). Através do exame de tomografia computadorizada que segundo Utumi et al. (2012) é indispensável para o diagnóstico, pois auxilia com maior precisão o planejamento cirúrgico notou-se uma imagem hipodensa, com deslocamento do dente 23 e destruição de cortical óssea vestibular.

Após analise clínica e radiográfica minuciosa é possível traçar um plano de tratamento baseado nas características particulares de apresentação da lesão cística. De forma geral o método de tratamento mais comum para o COC é a enucleação e curetagem com acompanhamento de longo prazo (Moreno-Rodriguez et al., 2020). Porém o emprego da marsupialização é encontrado na literatura como alternativa, pois essa técnica objetiva reduzir o tamanho de lesões com grandes dimensões através de descompressão passiva e com isso diminui a necessidade de uma intervenção cirúrgica mais complexa e radical, preservando estruturas importantes que podem estar associadas e/ou próximas ao cisto, essa regressão facilita a remoção total em posterior enucleação, diminui as chances de recidiva, além de iniciar o processo de reparação óssea (Moraes et al., 2020).

O diagnóstico definitivo é determinado através do exame anatomopatológico, que no presente caso corrobora com a literatura descrito de cavidade revestida por epitélio estratificado, camada basal com células colunares baixas semelhantes à pré-ameloblastos, camada intermediária de arranjo frouxo semelhante ao retículo estrelado do esmalte e em seu interior grupos de células anucleadas (células-fantasmas). Calcificações sobre o epitélio cístico e calcificações das células-fantasma também podem ser identificadas nos cortes histológicos (Neville, 2016; Mandú et al., 2016).

O acompanhamento no pós-operatório (PO) é de extrema importância para o manejo da patologia, sendo somada a colaboração do paciente para que através dos exames clínicos e radiográficos de controle seja possível escolher o momento para uma segunda intervenção pós marsupialização e retirada total da unidade cística (Moraes et al., 2020). Através dessa técnica, Souza et al. (2007) mostraram diminuição significativa no tamanho das lesões após 1 ano e 9 meses, já quando realizada enucleação e acompanhamento de longo prazo de dois casos afirma que não ocorreu recidiva após 10 anos no primeiro e 5 anos no segundo, enquanto Da Silva et al. (2014) relatou regressão parcial da entidade 5 meses após a descompressão passiva e sem recidiva após 13 meses da remoção total do cisto. Na tomografia realizada no pós-operatório de três meses foi possível notar diminuição de volume tridimensional e a presença de áreas de calcificação no interior da lesão, além de não recidiva nesse mesmo período.

\section{Conclusão}

O coc é uma lesão rara que pode surgir associada a um dente incluso sendo diferenciado de outras hipóteses diagnosticas através do exame histopatológico. Sendo uma lesão odontogênica rara dos ossos maxilares, a abordagem cirúrgica minimamente invasiva realizada, mostrou resultados positivos na diminuição e formação de áreas de calcificação da lesão, justificando a eleição da marsupialização como abordagem cirúrgica inicial para patologias desse gênero com grandes dimensões, trazendo benefícios para o paciente. 


\section{Referências}

Azambuja, T. W. F. et. al. (2005). Cisto Odontogênico calcificante: revisão da literatura e apresentação de caso clínico e cirurgia. Revista da faculdade de odontologia,5(2), 19-24.

Browne, R. H. M. A. R. M. (1982). The calcifying odontogenic cyst: a problem of preoperative diagnosis. British Journal of Oral Surgery, Birmingham Dental Hospital, 20(1), 203-212.

Cancio, A. V. et. al. (2005). Cisto odontogênico calcificante: relato de caso. Rev. cir. traumatol. buco-maxilo-fac. 6(1), 23-28.

Carvalhosa, A. A. et. al. (2005). Cisto de Gorlin: revisão de literatura. Pesqui. bras. odontopediatria clín. Integr. 5(3), 291-295.

Cavalcante, L. de L. et al. (2019). Cisto odontogênico calcificante na maxila posterior: um relato de caso atípico com acompanhamento de dois anos. SALUSVITA,38(2),409- 421.

Daniels, J. S. M. et al. (2014). Recurrent calcifying odontogenic cyst involving the maxillary sinus. Oral Surg Oral Med Oral Pathol Oral Radiol Endod, $98(1), 660-664$

Kusama, F. I. M. et.al. (2005). Cisto odontogênico calcificante mostrando um revestimento epitelial variado: um caso adicional com implicações para a capacitação de diferenciação divergente do epitélio do cisto. Head Neck Pathol.,13(2) 251-254.

Mandú, A. L. C. et al. (2016). Calcifying cystic odontogenic tumour associated with odontoma involving mixed dentition. Braz Dent Sci, 19 (3), $101-105$.

Medeiros, P. B. et.al. (2007). Cisto de Gorlin: relato de caso e revisão de literatura. Rev. cir. traumatol. buco-maxilo-fac. 7(1), 59-64.

Moleri, A. et al. (2002) Comparative Morphology of 7 New Cases of Calcifying Odontogenic Cysts. J Oral Maxillofac Surg, 60(1), 689-696.

Moraes, A. T. L. D. et al. (2020). Marsupialization before enucleation as a treatment strategy for a large calcifying odontogenic cyst: Case report. International Journal of Surgery Case Reports, 67(1), 239-244.

Olmedo-Cueva, S. et.al. (2020). Síndrome de Gorlin-Goltz. Diagnóstico molecular, nuevos tratamientos. Revista ADM. 77(3), $162-167$.

Pereira, A. S., et al. (2018). Metodologia da pesquisa científica. UFSM.

Pontes, H. A. R., (2009). Tumor odontogênico cístico calcificante em mandíbula: relato de caso e revisão de literatura. Rev para. Med. 23(1), 1-5.

Rodríguez, P. M. et al. (2020). Active decompression and distraction sugosteogenesis for the treatment of calcifying odontogenic cyst. Oral and Maxillofacial Surgery, 25(1), 89-97.

Silva, W. S., Adão, R. G. \& Moura, R. M. (2019). Cisto odontogênico calcificante: relato de caso. Barsilian Journal of Oral and maxilloofacial Sugery, 199(3), 25-29.

Silvaa, Y. S. D. et al. (2014). Effectiveness of surgical decompression in the treatment of a calcifying cystic odontogenic tumor. Autopsy and Case Reports, 4(4), 43-49.

Souza, L. N. et al. (2006). Conservative Treatment of Calcifying Odontogenic Cyst: Report of 3 Cases. J Oral Maxillofac Surg, 65(1), $2353-2356$.

Trejo, B. M. \& BELLO, L. C., (1999). Quiste odontogénico calcificante (quiste de Gorlin). Reporte de un caso y su seguimiento. Revisión de la literatura. Rev. ADM. 56(2), 83-7.

Utumi, E. R. et al. (2012). Distintas manifestações do tumor odontogênico cístico calcificante. Einstein, 10(3), 366-370. 